\title{
Assessing Thermochemical Properties of Materials through Ab initio Quantum-mechanical Methods: The Case of $\alpha-\mathrm{Al}_{2} \mathrm{O}_{3}$.
}

\author{
Alessandro Erba, ${ }^{a, *}$ Jefferson Maul, ${ }^{a, b}$ Raffaella Demichelis $^{c}$ and Roberto Dovesi $^{a}$ \\ Received Xth XXXXXXXXXX 20XX, Accepted Xth XXXXXXXXX 20XX \\ First published on the web Xth $X X X X X X X X X X 20 X X$ \\ DOI: 10.1039/b000000x
}

The thermochemical behavior of $\alpha-\mathrm{Al}_{2} \mathrm{O}_{3}$ corundum in the whole temperature range 0-2317 $\mathrm{K}$ (melting point) and under pressures up to $12 \mathrm{GPa}$ is predicted by applying $a b$ initio methods based on the density functional theory (DFT), the use of a local basis set and periodic-boundary conditions. Thermodynamic properties are treated both within and beyond the harmonic approximation to the lattice potential. In particular, a recent implementation of the quasi-harmonic approximation, in the CRYSTAL program, is here shown to provide a reliable description of the thermal expansion coefficient, entropy, constant-volume and constant-pressure specific heats, and temperature dependence of the bulk modulus, nearly up to the corundum melting temperature. This is a remarkable outcome suggesting $\alpha-\mathrm{Al}_{2} \mathrm{O}_{3}$ to be an almost perfect quasi-harmonic crystal. The effect of using different computational parameters and DFT functionals belonging to different levels of approximations on the accuracy of the thermal properties is tested, providing a reference for further studies involving alumina polymorphs and, more generally, quasiionic minerals.

\section{Introduction}

Aluminum oxide has been extensively used for decades in a wide variety of industrial applications due to its remarkable properties, including high melting point, density, electrical resistivity, hardness, resistance to weathering and low solubility. ${ }^{1}$ Corundum $\left(\alpha-\mathrm{Al}_{2} \mathrm{O}_{3}\right.$, the most stable form of aluminum oxide, whose atomic structure is sketched in Figure 1) and its polymorphs (the so-called transitions aluminas) also exhibit unique surface properties, which make them suitable both as catalysts for several reactions ${ }^{2-9}$ and as support for other catalysts. ${ }^{10-14}$ Furthermore, the thermodynamic properties of corundum are of particular interest in calorimetric studies in that it constitutes a standard reference material (SRM-720) for the calibration of some calorimeters. ${ }^{15}$

First-principles techniques based on the density functional theory (DFT) are becoming an important complementary tool in the investigation of mineral structure and thermodynamics, as well as surface properties and reactivity, as they allow to analyse the nature of the fundamental interactions giving rise to the observed phenomena. ${ }^{16}$ In this context, the ability

\footnotetext{
${ }^{a}$ Dipartimento di Chimica and Centre of Excellence Nanostructured Interfaces and Surfaces (NIS), Università di Torino, via Giuria 5, IT-10125 Torino, Italy. E-mail: alessandro.erba@unito.it

${ }^{b}$ Laboratório de Combustíveis e Materiais, INCTMN-UFPB, Universidade Federal da Paraíba, CEP 58051-900, João Pessoa, PB, Brazil

${ }^{c}$ Nanochemistry Research Institute, Curtin Institute for Computation, and Department of Chemistry, Curtin University, GPO Box U1987, Perth, WA 6845, Australia
}

to simulate realistic pressure and temperature conditions can make the difference between providing a qualitative or a quantitative prediction. In general, DFT modeling of solid state materials is performed at zero temperature and pressure, and thermodynamic functions are estimated through harmonic lattice dynamics under the assumption of no thermal expansion. This is certainly a rough approximation, though successful in many cases and frequently used. ${ }^{17-21}$ However, this approach is unable to provide accurate predictions related to high temperature conditions, as well as to phases whose structures and properties have a significant dependence on temperature and pressure. Several computational studies on aluminum oxide polymorphs have been reported in recent years, which mainly apply standard DFT methods. ${ }^{22-30}$

In this paper, we apply a recently developed technique based on the so-called quasi-harmonic approximation (QHA): a simple and effective method that is able to address the aforementioned issue in the case of corundum. ${ }^{31,32}$ The thermal expansion coefficient, $\alpha(T)$, heat capacities, $C_{V}(T)$ and $C_{P}(T)$, entropy, $S(T)$ and bulk modulus, $K(T)$ of corundum are calculated in its entire range of thermal stability (i.e. from $0 \mathrm{~K}$ up to its melting temperature, $T_{M}=2317 \mathrm{~K}$ ). The dependence of these properties on the level of approximation of the exchange-correlation functional, $V_{\mathrm{xc}}[\rho]$, is explicitly explored by considering six different expressions of $V_{\mathrm{xc}}[\rho]$, focusing on those ones that are widely adopted in calculating mineral properties (local density approximation, LDA; generalised gradient approximations, GGA; and hybrid functionals). The influ- 


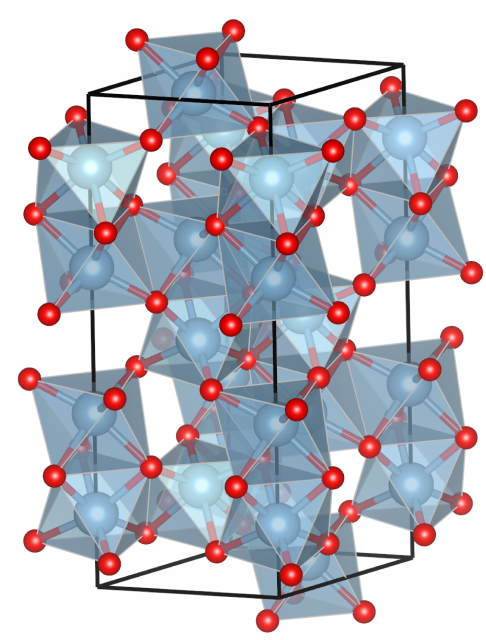

Fig. 1 (color online) Graphical representation of the atomic structure of corundum $\left(\alpha-\mathrm{Al}_{2} \mathrm{O}_{3}\right.$, space group $\left.R \overline{3} c\right)$ : aluminum is colored in gray, oxygen in red. $\mathrm{AlO}_{6}$ octahedra and lattice parameters of the conventional cell are also represented.

ence of other crucial computational parameters (e.g. supercell size in the calculation of phonon dispersion) on the calculated properties is also assessed.

One of the main reasons of interest in the study of minerals at simultaneous high-temperatures and high-pressures is the characterization of their elastic response at geochemical Earth mantle conditions. Indeed, a necessary prerequisite to the understanding of how seismic waves do propagate during earthquakes is precisely the knowledge of the elastic response of all possible constituents of the Earth mantle. Conversely, the only source of information on the actual composition of the Earth mantle are seismic data collected during earthquakes; different compositional models are used to interpret such data which strongly rely on the characterization of the elastic response of individual minerals at high temperature and pressure conditions. ${ }^{33-37}$ While a complete characterization of the thermo-elasticity of $\alpha-\mathrm{Al}_{2} \mathrm{O}_{3}$ would require the calculation of its fourth-rank elastic tensor at various temperatures and pressures (a quite demanding computational task which would give access to the full anisotropic description of the elastic response), ${ }^{38,39}$ the QHA offers a simplified approach to the investigation of the high-temperature, high-pressure behavior of an average elastic property of great geochemical interest: the bulk modulus $K$.

Estimations of some thermal properties of corundum, based on Kieffer's vibrational model upon experimental frequency data, have been reported in the literature, ${ }^{40,41}$ as well as a couple of DFT studies where the QHA was applied to the investigation of phase transitions in alumina polymorphs. ${ }^{29,30,42}$ Mousavi has recently reported some quasi-harmonic thermal properties of corundum, as computed with a simplified Debye model (where the lattice dynamics of the crystal is not explicitly solved at the $a b$ initio level of theory), which are found to largely disagree with available experimental data. ${ }^{43}$ To the best of our knowledge, the present investigation constitutes the first complete $a b$ initio description of thermodynamic and thermal structural and elastic properties of $\alpha-\mathrm{Al}_{2} \mathrm{O}_{3}$. This paper might then constitute a useful benchmark for undertaking future investigations involving aluminum oxide and related materials.

The structure of the paper is as follows: in Section 2 we briefly recall the main features of the adopted implementation of the quasi-harmonic approximation and we introduce the utilized computational setup; results on the thermal effects on structural and thermodynamic properties of $\alpha-\mathrm{Al}_{2} \mathrm{O}_{3}$ are discussed in Section 3 where the effect of the adopted functional of the DFT is explicitly investigated; the combined effect of pressure and temperature on such properties is also quantified; conclusions are drawn in Section 4.

\section{Computational Approach and Details}

All calculations are performed using a development version of the CRYSTAL program, ${ }^{44,45}$ where a fully-automated scheme for computing quasi-harmonic properties of crystals has recently been implemented, which relies on computing and fitting harmonic vibration frequencies at different volumes after having performed volume-constrained geometry optimizations. ${ }^{31,32}$ Such optimizations use analytical energy gradients with respect to both atomic coordinates and lattice parameters. Convergence is checked on energy, residual gradient components and magnitude of the nuclear displacements: default criteria are used. ${ }^{45}$ Harmonic phonon frequencies are computed by diagonalizing the dynamical matrix following a direct space approach. The influence of the adopted supercell size (equivalent to the sampling of the Brillouin zone in the reciprocal space approach) on computed thermodynamic properties will be analyzed in Section 3. Further details about the "direct space" approach to phonon dispersion calculation can be found elsewhere. ${ }^{31,46-48}$

Constant-volume specific heat, $C_{V}(T)$, and entropy, $S(T)$, are estimated through standard statistical thermodynamics from harmonic phonon frequencies as computed at the equilibrium zero temperature, zero pressure volume. Other properties (such as the constant-pressure specific heat, the thermal expansion coefficient or the temperature dependence of the bulk modulus), in order to be properly described, require to go beyond the simple harmonic approximation (HA) and are here computed by means of the QHA which is found to provide a satisfactory description of all of them nearly up to the melting temperature of corundum. The explored volume range extends from a $-4.3 \%$ compression to $\mathrm{a}+8.6 \%$ expan- 
sion with respect to the equilibrium volume and four volumes are explicitly considered in this interval.

The volumetric, isotropic, thermal expansion coefficient, $\alpha_{V}(T)$, is obtained by minimizing the isothermal Helmholtz free energy

$$
F^{\mathrm{QHA}}(T, V)=U_{0}^{\mathrm{ZP}}(V)+k_{B} T \sum_{\mathbf{k} p}\left[\ln \left(1-e^{-\frac{\hbar \omega_{\mathbf{k} p}(V)}{k_{B} T}}\right)\right]
$$

with respect to volume at several temperatures, where $k_{B}$ is Boltzmann's constant and $U_{0}^{\mathrm{ZP}}(V)$ is the zero-temperature internal energy of the crystal which includes the zero-point energy of the system: $E_{0}^{\mathrm{ZP}}(V)=\sum_{\mathbf{k} p} \hbar \omega_{\mathbf{k} p}(V) / 2$. The scheme, recently developed and implemented into the CRYSTAL program, ${ }^{31,32}$ is here generalized to the case of anisotropic thermal expansions: directional thermal expansion coefficients, $\alpha_{a}(T)$ and $\alpha_{c}(T)$, corresponding to the $a$ and $c$ symmetryindependent lattice parameters of $\alpha-\mathrm{Al}_{2} \mathrm{O}_{3}$ are computed which allow for a finer description of the thermal expansion mechanism.

One of the powerful advantages of the QHA is that of allowing for a natural combination of pressure and temperature effects on structural and elastic properties of materials. By differentiating equation (1) with respect to the volume and changing sign, the thermal pressure is obtained:

$$
P(V ; T)=-\frac{\partial F^{\mathrm{QHA}}(V ; T)}{\partial V} .
$$

The description of the isothermal bulk modulus of the system at simultaneous high-temperatures and high-pressures, $K_{T}(P, T)$, can be obtained as an isothermal second derivative of equation (1) with respect to the volume and by exploiting relation (2):

$$
K_{T}(P, T)=V(P, T)\left(\frac{\partial^{2} F^{\mathrm{QHA}}(V(P, T) ; T)}{\partial V(P, T)^{2}}\right)_{T} .
$$

Let us finally recall that the QHA allows for computing the difference between constant-pressure and constant-volume specific heats as follows: ${ }^{49,50}$

$$
C^{Q H A} \equiv C_{P}(T)-C_{V}(T)=\alpha_{V}^{2}(T) K(T) V(T) T .
$$

All-electron atom-centered Gaussian-type-function basis sets, of TZVP quality, are adopted. ${ }^{51}$ Six different formulations of the exchange-correlation functional of the DFT are considered, corresponding to some of the most widely used schemes within the LDA $\left(\mathrm{SVWN}^{52,53}\right)$, GGA (PBE, ${ }^{54}$ PW91, ${ }^{55}$ $\mathrm{PBEsol}^{56}$ ) and hybrid approaches (B3LYP, ${ }^{57} \mathrm{PBE0}^{58}$ ). Thresholds controlling the accuracy of Coulomb and exchange series are set to $10^{-8}\left(T_{1}\right.$ to $\left.T_{4}\right)$ and $10^{-16}\left(T_{5}\right)$ for the integral truncation and to $10^{-22}$ and $10^{-18}$ for the selection of bi-electronic integrals that can be approximated by the bipolar expansion. ${ }^{45}$ Reciprocal space is sampled using a MonkhorstPack mesh with a shrinking factor of 8,4 and 2 when performing the calculation on the primitive cell, the conventional cell or largest cells (like a $2 \times 2 \times 2$ or a $3 \times 3 \times 3$ expansion of the primitive cell), corresponding to 65,14 and 4 independent $k$-points in the irreducible portion of the Brillouin zone. A pruned grid with 1454 radial and 99 angular points is used to calculate the DFT exchange-correlation contribution through numerical integration of the electron density over the unit cell volume. ${ }^{45}$ The Self-Consistent-Field (SCF) convergence on energy was set to a value of $10^{-10}$ hartree for all geometry optimizations and phonon frequency calculations.

\section{Results and Discussion}

\subsection{Thermodynamic Properties}

When computing thermodynamic properties of crystals, one has to make sure that the description of the lattice dynamics of the system is properly converged with respect to the sampling of the dispersion of phonon branches in reciprocal space. Equivalently, given the direct space nature of the approach here utilized, convergence of computed properties with the size of the adopted supercell (SC) has to be carefully checked for. If harmonic thermodynamic functions, such as entropy and constant-volume specific heat, show a rather slow convergence (more so for $S$ than $C_{V}$ ), the situation is generally much more favorable for quasi-harmonic ones. For instance, some of us have recently shown that for fully ionic systems, such as $\mathrm{MgO}$ and $\mathrm{CaO}$, a $\mathrm{SC}$ containing 128 atoms is required to converge the entropy while a SC containing just 8 atoms already provides a converged description of all quasi-harmonic quantities. ${ }^{32}$ Figure 2 shows such a convergence for entropy and constant-volume specific heat of $\alpha-\mathrm{Al}_{2} \mathrm{O}_{3}$, as computed at the PBE0 level. Four SCs of increasing size are considered, containing 10, 30, 80 and 270 atoms and corresponding to the primitive cell, the conventional cell, a $2 \times 2 \times 2$ expansion of the primitive cell and a $3 \times 3 \times 3$ expansion of the primitive cell. It is seen that both quantities are practically converged with a SC containing 80 atoms, more so for $C_{V}$ than for $S$ : at $T_{M}$, the difference between the 80 and the 270 atom SCs being just $0.9 \%$ and $1.3 \%$ for $C_{V}$ and $S$, respectively. The SC containing 270 atoms is then expected to give results that are most likely to be fully converged. In the figure, we also report accurate experimental data to compare with: empty circles refer to $C_{V}$ values by Saxena and Shen ${ }^{59}$ while full circles to $S$ values by Robie et $a l .{ }^{60}$ For both quantities, the agreement between computed and experimental data is rather satisfactory in the whole temperature range and makes $a b$ initio simulations a reliable predictive tool for these harmonic properties. Analogously to what recently documented for fully ionic systems, ${ }^{32}$ also in the present case of a mixed ionic/covalent system, the effect 

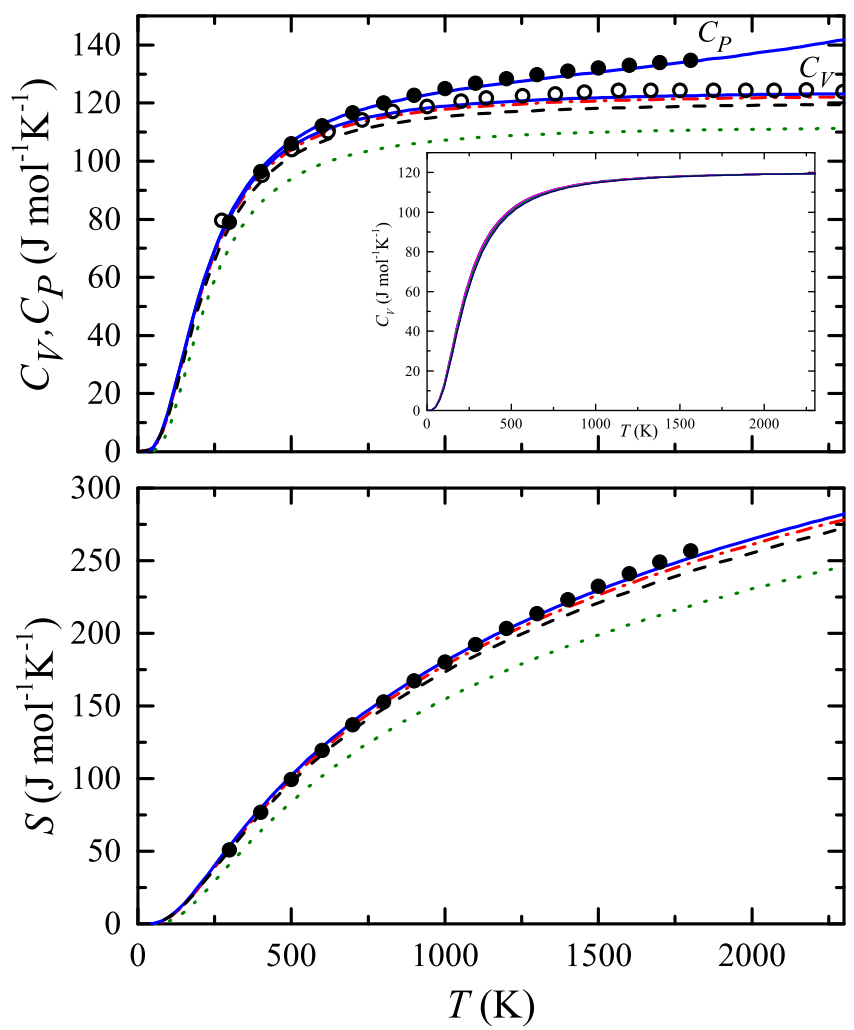

Fig. 2 (color online) Specific heat, $C_{V}$, (upper panel) and entropy $S$ (lower panel) of $\alpha-\mathrm{Al}_{2} \mathrm{O}_{3}$ as a function of temperature, as computed at PBE0 level with SCs of increasing size: 10 (dots), 30 (dashed), 80 (dot-dashed) and 270 (continuous) atoms. The uppermost line of the top panel is the $C_{P}$ curve obtained by adding the $C^{Q H A}$

quasi-harmonic contribution (evaluated according to equation 4 ) to the converged $C_{V}$ one. The inset of the upper panel shows the $C_{V}$ as computed with all the six DFT functionals considered in this study for the SC containing 30 atoms. Empty circles refer to experimental $C_{V}$ values from Saxena and Shen, ${ }^{59}$ full circles are experimental $S$ and $C_{P}$ data from Robie et al. ${ }^{60}$

of the adopted DFT functional on such quantities is almost negligible as they are both dominated by acoustic modes generally correctly described by all functionals. In this respect, the inset of the upper panel of Figure 2 shows $C_{V}$ as computed with the six DFT functionals considered in this study (LDA, PBE, PBEsol, PW91, B3LYP and PBE0) for the 30 atom SC; the six curves are essentially superimposed to each other. In the upper panel of the figure, the constant-pressure specific heat is also reported which will be commented on later as it is a quasi-harmonic and not a simple harmonic quantity.

The effect of the SC size on the convergence of quasiharmonic properties is shown in Figure 3 where the differences between thermal expansion coefficients, $\Delta \alpha$, and quasiharmonic contribution to the specific heat, $\Delta C^{Q H A}$, as computed with SCs of different size and those obtained with the

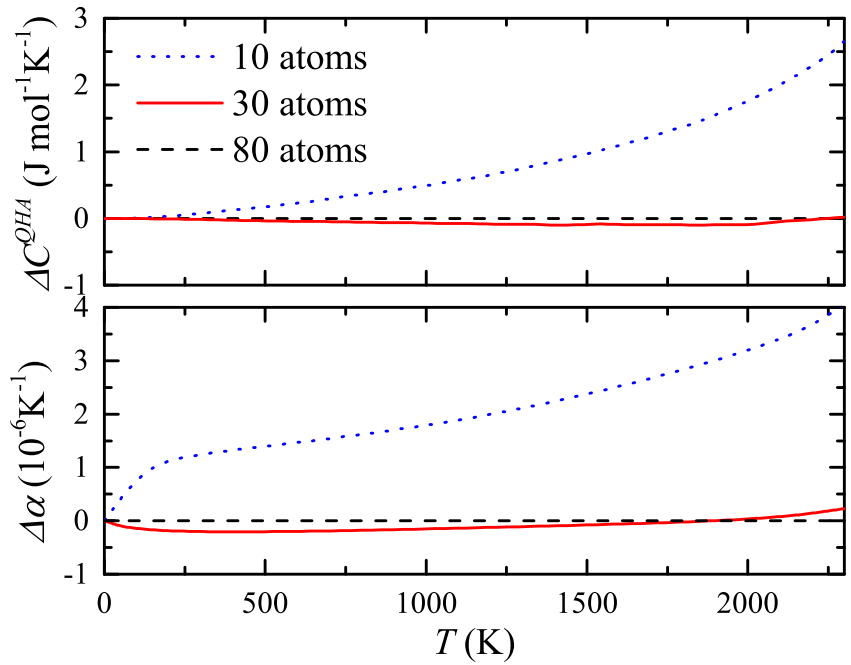

Fig. 3 (color online) Convergence of thermal expansion coefficient $\alpha_{V}(T)$ and $C^{Q H A}$ (see equation 4) of $\alpha-\mathrm{Al}_{2} \mathrm{O}_{3}$ as a function of the adopted SC size. Differences, $\Delta \alpha$ and $\Delta C^{Q H A}$, are reported with respect to the SC containing 80 atoms. All results are obtained with the PBE0 functional.

SC containing 80 atoms are reported. It is clearly seen that if the primitive cell (10 atoms) is not a suitable model, the conventional SC containing just 30 atoms provides a description of both quantities which is essentially converged, with an average deviation of $0.3 \%$ for $C^{Q H A}$ and $0.9 \%$ for $\alpha_{V}(T)$ over the whole temperature range with respect to the SC with 80 atoms. The effect of the adopted DFT functional on the quasiharmonic description will be discussed in Section 3.2. By adding the converged $C^{Q H A}$ quasi-harmonic term to the converged $C_{V}$ harmonic contribution, the final constant-pressure specific heat, $C_{P}$, is obtained which is reported in the upper panel of Figure 2 as a continuous line and compared with available experimental data by Robie et al. ${ }^{60}$ (full circles). This comparison shows how for $\alpha-\mathrm{Al}_{2} \mathrm{O}_{3}$ the QHA is capable of correctly describing $C_{P}(T)$ up to its melting point, which is a remarkable result given that for most systems the validity domain of the QHA is restricted to temperatures well-below the melting one. ${ }^{32,61}$ This is a first evidence, to be further confirmed below, of the fact that $\alpha-\mathrm{Al}_{2} \mathrm{O}_{3}$ corundum essentially behaves as an almost perfect quasi-harmonic crystal, with no particular need for an explicit account of intrinsic anharmonic effects beyond the QHA.

\subsection{Structural Properties}

The thermal behavior of structural and elastic properties of $\alpha$ $\mathrm{Al}_{2} \mathrm{O}_{3}$ is now discussed as determined at the quasi-harmonic level of theory. The effect on computed properties of the particular choice of the adopted DFT functional (three different 

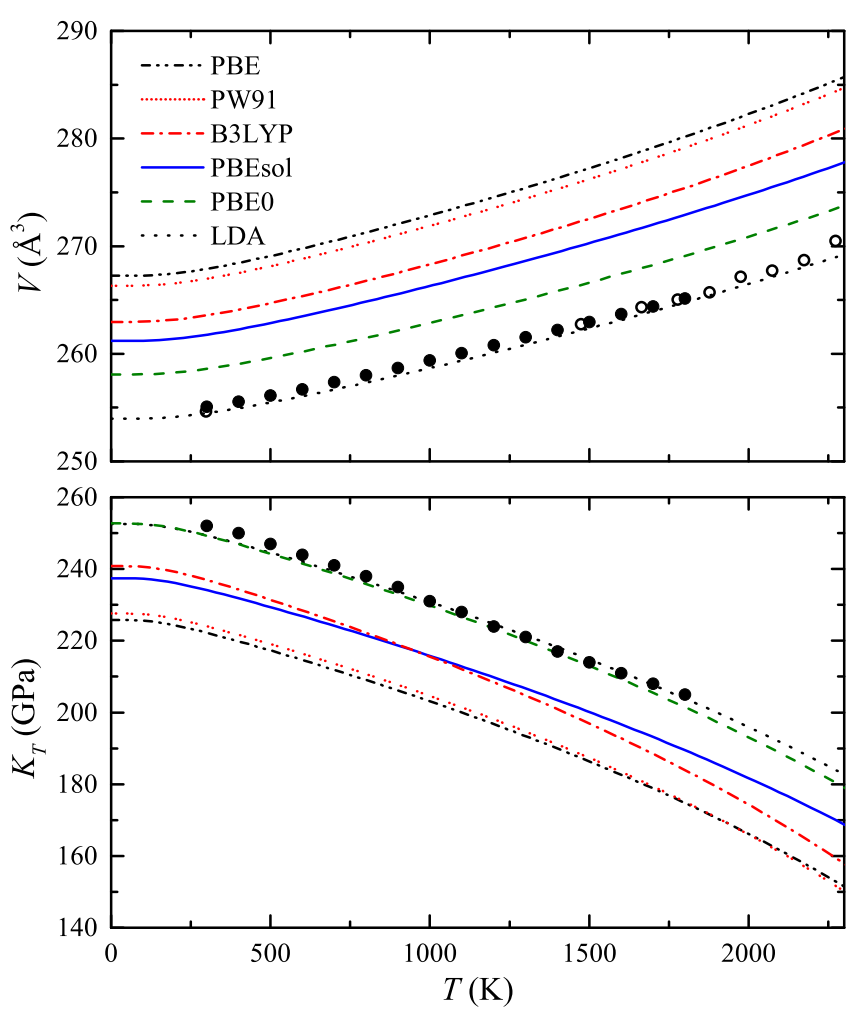

Fig. 4 (color online) Dependence of the conventional cell volume, $V$, and bulk modulus, $K$, of $\alpha-\mathrm{Al}_{2} \mathrm{O}_{3}$ on temperature, as computed with different DFT functionals at the quasi-harmonic level. Full and empty circles correspond to experimental data by Anderson et al. ${ }^{62}$ and Fiquet et al. ${ }^{63}$ respectively.

classes of functionals, LDA, GGA and hybrids, are explicitly considered) is discussed, which may serve as a benchmark for future studies on aluminum oxides. In Figure 4, we report the equilibrium volume (of the conventional cell) and the bulk modulus of corundum as a function of temperature, up to its melting point. Experimental data are by Anderson et al. ${ }^{62}$ (full circles) and by Fiquet et al. ${ }^{63}$ (empty circles). From the analysis of the figure, some considerations can be made: i) different functionals do provide very different values of the equilibrium volume and bulk modulus, with differences among them as large as $5 \%$ for the volume and $11 \%$ for the bulk modulus; ii) as already observed in previous studies, the LDA functional provides the lowest volume and the highest bulk modulus among them while pure GGA functionals such as PBE and PW91 give the highest volumes and lowest bulk moduli; iii) despite the very different description of the absolute value of these properties, all functionals provide a fairly correct description of the temperature dependence of $V$ and $K$ almost up to $T_{M}$, with small differences among them to be discussed below; iv) surprisingly enough, in this case, the simple LDA functional provides results in
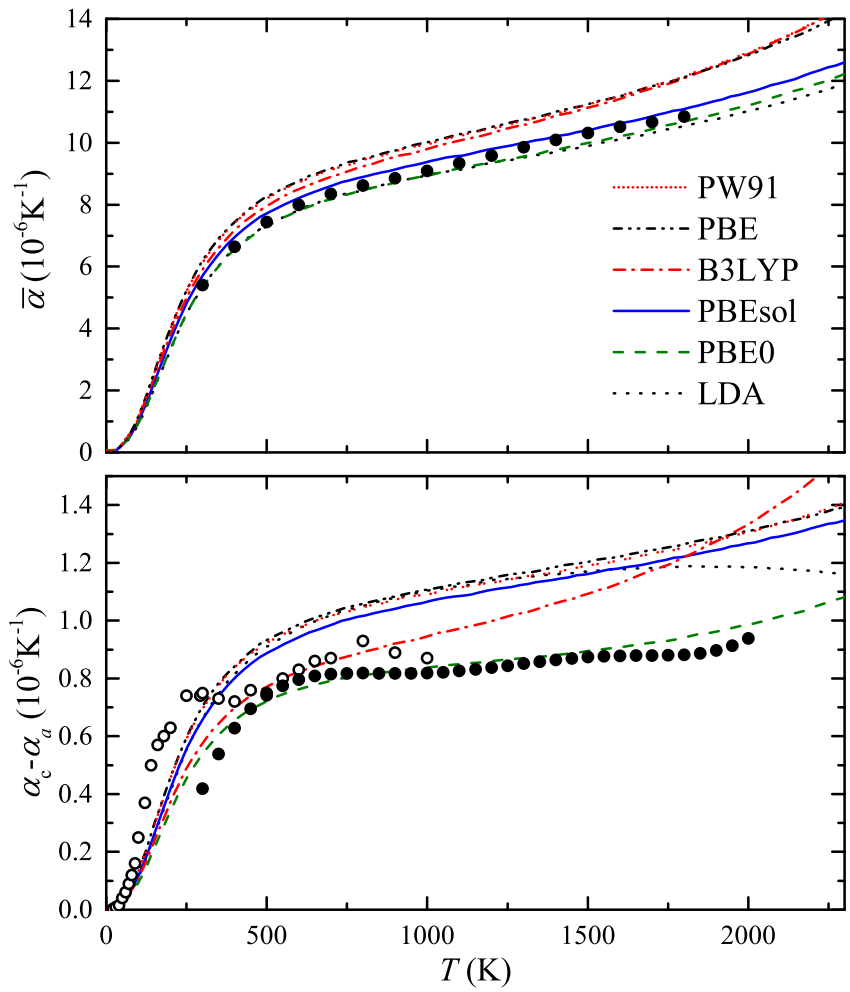

Fig. 5 (color online) Mean isotropic $\left(\bar{\alpha}=\alpha_{V} / 3\right)$ and anisotropic $\left(\alpha_{c}-\alpha_{a}\right)$ thermal expansion coefficient of $\alpha-\mathrm{Al}_{2} \mathrm{O}_{3}$ calculated as a function of temperature with various DFT functionals. Full circles represent experimental data by Anderson et al. ${ }^{62}$ for $\alpha_{V} / 3$ and by Munro $^{72}$ for $\alpha_{c}-\alpha_{a}$; empty circles are data from White and Roberts. ${ }^{73}$

excellent agreement with the experimental data, followed by the PBE0 hybrid functional; PBE and PW91, on the contrary, give the poorest description among those explored; v) PBEsol, the PBE re-parametrization specifically designed for the solid state, significantly improves the description of both quantities, even if not to a sufficient extent to bring its results close to the experimental ones; vi) the inclusion of the zero-point motion on zero-temperature values increases the volume by about $1 \%$ and decreases the bulk modulus by about $1.5 \%$ for all functionals (not shown in the figure). The exceptional performance of LDA in reproducing equilibrium structural features of $\alpha$ $\mathrm{Al}_{2} \mathrm{O}_{3}$ has already been discussed in several ab initio studies. ${ }^{30,64-71}$

The good description of the temperature dependence of $V(T)$ and $K(T)$ up to $T_{M}$, without strong deviations which are commonly seen in other systems (particularly on $K(T)$ ) well below $T_{M},{ }^{32}$ is a strong evidence of the quasi-harmonic character of $\alpha-\mathrm{Al}_{2} \mathrm{O}_{3}$ corundum.

Let us now analyze the description of the thermal expansion of $\alpha-\mathrm{Al}_{2} \mathrm{O}_{3}$ into more detail. The upper panel of Fig- 
ure 5 shows the average isotropic thermal expansion coefficient, $\bar{\alpha}=\alpha_{V} / 3$, of corundum as computed with the six DFT functionals here considered. Accurate experimental data by Anderson et al. ${ }^{62}$ are reported as full circles. Despite an overall good description of the thermal expansion of corundum by all functionals, this figure allows for a finer discussion. It can be seen that three functionals (LDA, PBEsol and PBE0) perform better than the other three (PBE, PW91 and B3LYP) that slightly overestimate $\bar{\alpha}$. An interesting feature of the thermal expansion of non-cubic crystals is its anisotropy. If one considers the conventional cell of $\alpha-\mathrm{Al}_{2} \mathrm{O}_{3}$, there are two symmetry-independent lattice parameters: $a \equiv b$ and $c$; the corresponding directional thermal expansion coefficients, $\alpha_{a}(T)$ and $\alpha_{c}(T)$, can be computed (the relation $\alpha_{V}=2 \alpha_{a}+\alpha_{c}$ holds true). In the lower panel of Figure 5 we report the $\alpha_{c}-\alpha_{a}$ difference as a function of temperature. Experimental data by Munro ${ }^{72}$ (full circles) and White and Roberts ${ }^{73}$ (empty circles) are also reported, the latter being not as smooth as the former ones. Some remarks can be made: i) the $\alpha_{c}-\alpha_{a}$ difference is positive both experimentally and theoretically at all levels of theory thus implying a larger thermal expansion along the $c$ crystallographic axis than in the basal plane $a b$; ii) the anisotropy of the thermal expansion amounts to about $10 \%$ of the total; iii) the steep behavior of experimental data by White and Roberts ${ }^{73}$ at temperatures below $250 \mathrm{~K}$ seems to be inconsistent with all theoretical predictions and other experimental data by Munro; ${ }^{72}$ iv) the PBE0 hybrid functional describes an anisotropy which is very close to the experimental one by Munro, ${ }^{72}$ B3LYP provides a reasonable description up to about $600 \mathrm{~K}$ and then rapidly increases, while the other functionals seem to systematically overestimate it by about $20 \%$.

\subsection{Effect of Thermal Pressure}

While the inclusion of pressure on computed properties of solids can be considered a relatively simple task, ${ }^{37,77-82}$ the combined description of temperature and pressure is much more demanding and seldom reported in $a b$ initio studies. As recalled in Section 2, one of the advantages of the quasiharmonic approximation is precisely that of allowing for a natural combination of both temperature and pressure on thermodynamic and structural properties of solids. The so-called thermal pressure can indeed be computed as a volume derivative of the isothermal Helmholtz free energy (see equation 2). The pressure-volume-temperature $(P-V-T)$ equation-of-state (EOS) is then easily obtained. In Figure 6, we report two different representations (which correspond to constant-pressure and constant-temperature experiments) of the $P-V-T$ EOS of $\alpha-\mathrm{Al}_{2} \mathrm{O}_{3}$, as computed with the hybrid PBE0 functional that has been found to reliably describe most of the properties discussed in the previous paragraphs. In the left panel of the

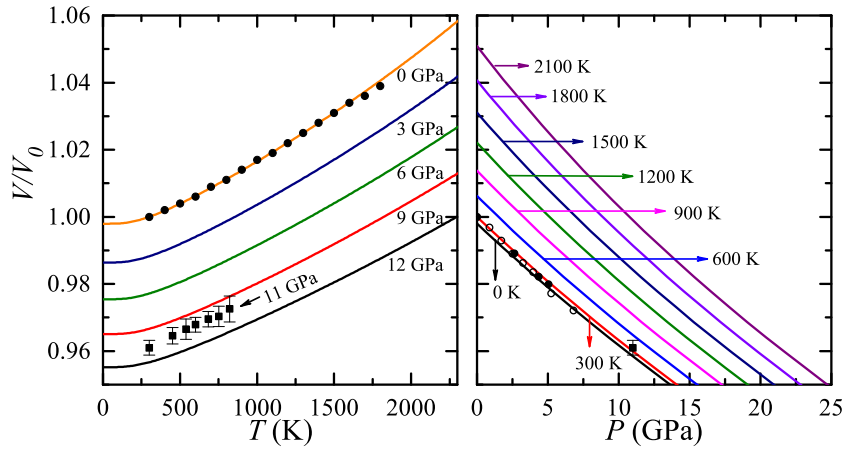

Fig. 6 (color online) $V / V_{0}$ as a function of temperature at 5 different pressures (left panel) and as a function of pressure at 8 different temperatures (right panel), where $V_{0}$ is the equilibrium volume at $300 \mathrm{~K}$ and zero pressure of $\alpha-\mathrm{Al}_{2} \mathrm{O}_{3}$. Full circles are experimental data from Anderson et al. ${ }^{62}$ (in the left panel) and from Sato and Akimoto ${ }^{74}$ (in the right panel), empty circles from Grevel et al. ${ }^{75}$ while full squares and corresponding error bars are from Dubrovinsky et al. ${ }^{76}$ All computed values are obtained at the PBE0 level.

figure, the $V / V_{0}$ ratio (where $V_{0}$ is the equilibrium volume at $300 \mathrm{~K}$ and zero pressure) is reported as a continuous function of temperature, up to corundum melting point, at five equidistant pressures $(0,3,6,9$ and $12 \mathrm{GPa})$. Experimental data at zero pressure by Anderson et al. ${ }^{62}$ are given as full circles while those at about $11 \mathrm{GPa}$ by Dubrovinsky et al. ${ }^{76}$ as full squares, with corresponding error bars. The effect of pressure is a non-linear compression of the unit cell volume. The compression of the cell volume due to pressure is more pronounced at higher temperatures than at lower temperatures so that, as a result, pressure clearly reduces the thermal expansivity of $\alpha-\mathrm{Al}_{2} \mathrm{O}_{3}$ (see below). In the right panel of the figure, we report the same quantity, $V / V_{0}$, as a function of pressure at eight equidistant temperatures $(0,300,600,900$, $1200,1500,1800$ and $2100 \mathrm{~K})$. Experimental data are also reported as measured at $300 \mathrm{~K}$ at different pressures by Sato and Akimoto $^{74}$ (full circles), Grevel et al. ${ }^{75}$ (empty circles) and Dubrovinsky et al. ${ }^{76}$ (full square). We can clearly see that: i) obviously as temperature increases the cell volume expands; ii) a given volume (i.e. a given value of the $V / V_{0}$ ratio) corresponds to higher pressures as temperature increases.

As we have just anticipated, from inspection of the left panel of Figure 6 one can see how pressure is progressively reducing the thermal expansivity of corundum. In order to give a more quantitative representation of such effect we introduce the upper panel of Figure 7 where the volumetric thermal expansion coefficient, $\alpha_{V}$, of $\alpha-\mathrm{Al}_{2} \mathrm{O}_{3}$ is plotted as a function of temperature at five equidistant pressures. Experimental data at zero pressure, from Anderson et al., ${ }^{62}$ are in excellent agreement with PBE0 theoretical ones, as already noticed while dis- 

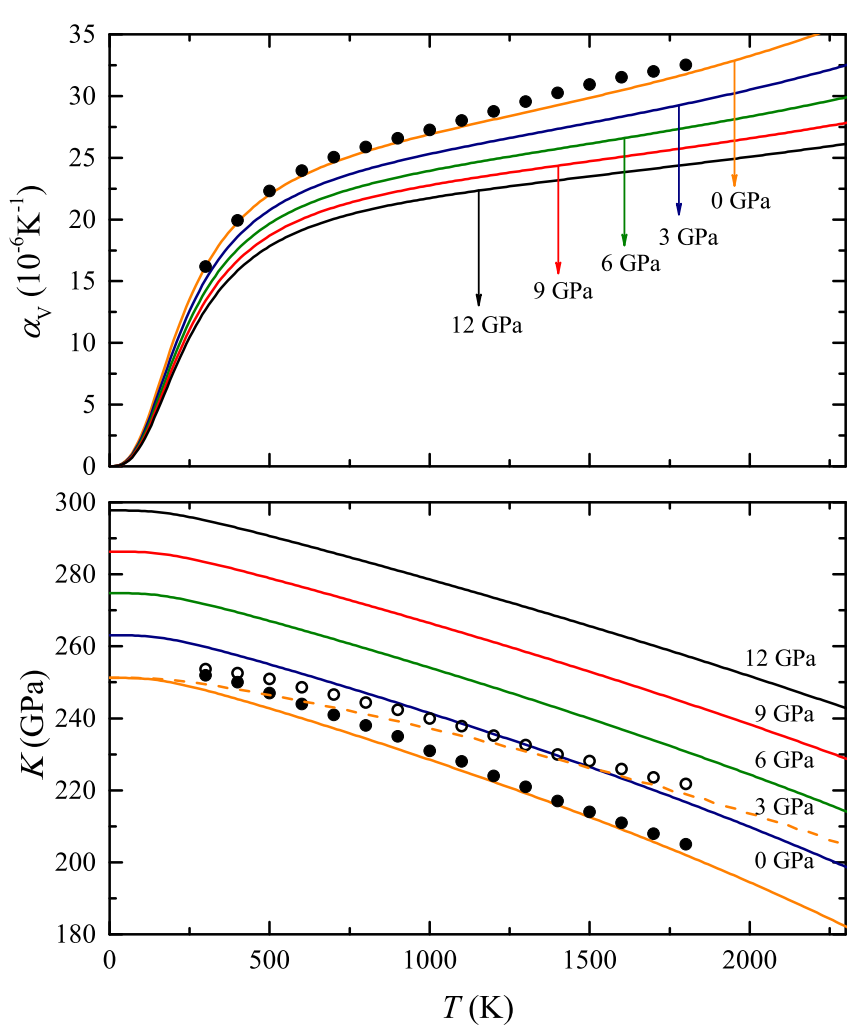

Fig. 7 (color online) Dependence on temperature, up to $2300 \mathrm{~K}$, and pressure, up to $12 \mathrm{GPa}$, of the thermal expansion coefficient (upper panel) and bulk modulus (lower panel) of $\alpha-\mathrm{Al}_{2} \mathrm{O}_{3}$, as computed at PBE0 level. Experimental data at ambient pressure from Anderson et al. ${ }^{62}$ are reported for comparison. In the lower panel, full circles refer to the isothermal bulk modulus, $K_{T}$, while empty circles to the adiabatic bulk modulus, $K_{S}$. In the lower panel, the dashed line represents the computed adiabatic bulk modulus at zero pressure.

cussing Figure 5. It is clearly seen that, as pressure linearly increases, the thermal expansivity of corundum decreases (not linearly). As we could not find any high-pressure experimental data of the thermal expansion coefficient of $\alpha-\mathrm{Al}_{2} \mathrm{O}_{3}$ and given the excellent description provided by the hybrid PBE0 functional so far, these values are expected to constitute reliable predictions of the high-temperature, high-pressure behavior of corundum.

As recalled in the Introduction, the description of the bulk modulus of minerals at simultaneous high temperature and pressure conditions is of particular geochemical interest. In the lower panel of Figure 7, we report the isothermal bulk modulus, $K_{T}$, computed as the volume second derivative of the isothermal Helmholtz free energy (see equation 3 ). It is reported as a continuous function of temperature at five distinct pressures, up to $12 \mathrm{GPa}$. Pressure is seen to systematically increase the bulk modulus, more so at higher temper- atures. Isothermal experimental determinations at zero pressure from Anderson et al. ${ }^{62}$ are reported as full circles. The agreement with the PBE0 theoretical description (lowest continuous line) is remarkable in the whole temperature range. Some experimental techniques for measuring the bulk modulus of a crystal involve elastic waves and are characterized by very short time-scales that prevent the system from reaching a thermal equilibrium; in these cases, an adiabatic bulk modulus, $K_{S}$, is measured. Adiabatic and isothermal bulk moduli do coincide with each other at zero temperature only, $K_{S}$ always being larger than $K_{T}$ at any finite temperature. In the figure, for instance, empty circles do represent adiabatic bulk modulus values as measured at zero pressure by Goto et al. ${ }^{83}$ with the rectangular parallelepiped resonance method. The QHA also offers a way to compute the adiabatic bulk modulus from the isothermal one, given that:

$$
K_{S}=K_{T}+\frac{\alpha_{V}^{2} V T K_{T}^{2}}{C_{V}}=K_{T} \times \frac{C_{P}}{C_{V}},
$$

where the dependence of all quantities on temperature is omitted for clarity sake and in the last equality equation (4) has been exploited. The computed adiabatic bulk modulus at zero pressure is reported in the figure as a dashed line; again, the agreement with the experimental determinations is quite remarkable and confirms once more how the QHA allows for accurately describing structural and elastic features of $\alpha-\mathrm{Al}_{2} \mathrm{O}_{3}$ up to the melting temperature.

\section{Conclusions}

The dependence on temperature of a variety of thermodynamic, structural and elastic properties of $\alpha-\mathrm{Al}_{2} \mathrm{O}_{3}$ has been accurately determined by means of $a b$ initio simulations exploiting both the harmonic and quasi-harmonic approximations. The validity domain of the quasi-harmonic approximation, being often limited to temperatures much lower than the melting point $T_{M}$, is here found to extend almost up to $T_{M}$ thus suggesting that corundum essentially behaves as a perfect quasi-harmonic crystal with negligible intrinsic anharmonic effects. As other aluminum oxide polymorphs exhibit analogous structural and chemical features, from present results, the quasi-harmonic approximation is expected to constitute an effective and reliable approach to their thermal characterization.

The effect of the adopted functional of the density functional theory on thermal properties of $\alpha-\mathrm{Al}_{2} \mathrm{O}_{3}$ has been explicitly investigated and will prove a useful benchmark for future theoretical studies requiring a good thermodynamic description of aluminum oxides. Six different formulations, belonging to three different rungs of the "Jacob's ladder", have been considered: i) all functionals accurately describe 
harmonic thermodynamic properties such as entropy and constant-volume specific heats; ii) the simple LDA functional fortuitously provides an excellent description of the absolute values of both cell volume and bulk modulus of corundum; iii) all functionals are seen to correctly describe isotropic thermal properties up to $T_{M}$; iv) the hybrid PBE0 functional gives the best description of the thermal expansion coefficient (including its anisotropy which is overestimated by about $20 \%$ by the other functionals).

The combined effect of temperature and pressure, up to $12 \mathrm{GPa}$, has been predicted for structural and elastic properties which has a particular relevance to the field of geochemistry where such characterizations are extremely useful in finetuning and validating different compositional models for the Earth mantle.

\section{Acknowledgements}

J.M. acknowledges the Brazilian scholarship program "Ciência sem Fronteiras" (Process Number 248425/20137/SWE). RDe acknowledges Curtin University for funding through the Curtin Research Fellowship scheme, iVEC facilities and the Australian National Computing Infrastructure for the provision of computer time.

\section{References}

1 L. D. Hart, Alumina Chemicals: Science And Technology Handbook (The American Ceramic Society, Westerville, Ohio, 1990).

2 S. D. Chakarova-Käck, Ø. Borck, E. Schröder, and B. I. Lundqvist, Phys. Rev. B 74, 155402 (2006).

$3 \varnothing$. Borck, P. Hyldgaard, and E. Schröder, Phys. Rev. B 75, 035403 (2007).

4 V. A. Ranea, I. Carmichael, and W. F. Schneider, J. Phys. Chem. C 113, 2149 (2009).

5 Y. Zhao, Z. Chen, and J. Zhao, Environ. Sci. Technol. 44, 2035 (2010).

6 J. G. Catalano, J. Phys. Chem. C 114, 6624 (2010).

7 D. P. Woodruff, Chem. Rev. 113, 3863 (2013).

8 J. H. Park, K. Kim, Y. B. Yoo, S. Y. Park, K.-H. Lim, K. H. Lee, H. K. Baik, and Y. S. Kim, J. Mater. Chem. C 1, 7166 (2013).

9 M. Ruan, H. Hou, W. Li, and B. Wang, J. Phys. Chem. C 118, 20889 (2014).

10 T. J. Godin and J. P. LaFemina, Phys. Rev. B 49, 7691 (1994).

11 P. Bera, K. C. Patil, V. Jayaram, M. S. Hegde, and G. N. Subbanna, J. Mater. Chem. 9, 1801 (1999).

12 T. K. Todorova, M. V. Ganduglia-Pirovano, and J. Sauer, J. Phys. Chem. C 111, 5141 (2007).
13 A. Hellman and H. Grönbeck, J. Phys. Chem. C 113, 3674 (2009).

14 J. L. Achtyl, I. V. Vlassiouk, S. Dai, and F. Geiger, J. Phys. Chem. C 118, 17745 (2014).

15 D. G. Archer, J. Phys. Chem. Ref. Data 22, 1441 (1993).

16 G. E. Brown, Jr and G. Calas, Mineral-Acqueous Solution Interfaces and Their Impact on the Environment (European Association of Geochemistry, 2012-2013), vol. 1(6) of Geochemical Perspectives, chap. 6, pp. 540-544.

17 R. Demichelis, P. Raiteri, J. D. Gale, and R. Dovesi, J. Phys. Chem. C 117, 17814 (2013).

18 A. M. Ritzmann, A. B. Muñoz-García, M. Pavone, J. A. Keith, and E. A. Carter, Chem. Mater. 25, 3011 (2013).

19 M. Digne, P. Sautet, P. Raybaud, P. Euzen, and H. Toulhoat, J. Catal. 211, 1 (2002).

20 C. Wolverton and K. C. Hass, Phys. Rev. B 63, 024102 (2001).

21 R. Demichelis, Y. Noël, P. Ugliengo, C. M. ZicovichWilson, and R. Dovesi, J. Phys. Chem. C 115, 13107 (2011).

22 M. F. Peintinger, M. J. Kratz, and T. Bredow, J. Mater. Chem. A 2, 13143 (2014).

23 J. Sarsam, M. W. Finnis, and P. Tangney, J. Chem. Phys. 139, 204704 (2013).

24 C. K. Narula and G. M. Stocks, J. Phys. Chem. C 116, 5628 (2012).

25 S. Aryal, P. Rulis, L. Ouyang, and W. Y. Ching, Phys. Rev. B 84, 174123 (2011).

26 A. R. Ferreira, E. Küçükbenli, A. A. Leitão, and S. de Gironcoli, Phys. Rev. B 84, 235119 (2011).

27 B. Xu, H. Stokes, and J. Dong, J. Phys. Condens. Matter 22, 315403 (2010).

28 K. Jiang, D. Music, K. Sarakinos, and J. Schneider, J. Phys. Condens. Matter 22, 505502 (2010).

29 K. Umemoto and R. M. Wentzcovitch, Proc. Natl. Acad. Sci. U.S.A. 105, 6526 (2008).

30 A. R. Oganov and S. Ono, Proc. Natl. Acad. Sci. U.S.A. 102, 10828 (2005).

31 A. Erba, J. Chem. Phys. 141, 124115 (2014).

32 A. Erba, M. Shahrokhi, R. Moradian, and R. Dovesi, J. Chem. Phys. 142, 044114 (2015).

33 A. E. Ringwood, Composition and Petrology of the Earth's Mantle (McGraw-Hill, New York, 1975).

34 J. D. Bass and D. L. Anderson, Geophys. Res. Lett. 11, 229 (1984).

35 D. L. Anderson and J. D. Bass, Nature 320, 321 (1986).

36 A. Erba, A. Mahmoud, R. Orlando, and R. Dovesi, Phys. Chem. Miner. 41, 151 (2014).

37 A. Erba, A. Mahmoud, D. Belmonte, and R. Dovesi, J. Chem. Phys. 140, 124703 (2014). 
38 B. B. Karki, R. M. Wentzcovitch, S. de Gironcoli, and S. Baroni, Science 286, 1705 (1999).

39 B. B. Karki, R. M. Wentzcovitch, S. de Gironcoli, and S. Baroni, Phys. Rev. B 61, 8793 (2000).

40 P. F. McMillan and N. L. Ross, Phys. Chem. Miner. 14, 225 (1987).

41 J.-A. Xu, E. Huang, J.-F. Lin, and L. Y. Xu, Am. Mineral. 80, 1157 (1995).

42 Z. Łodziana and K. Parliński, Phys. Rev. B 67, 174106 (2003).

43 S. Mousavi, Optoelectron. Adv. Mat. 8, 1191 (2014).

44 R. Dovesi, R. Orlando, A. Erba, C. M. Zicovich-Wilson, B. Civalleri, S. Casassa, L. Maschio, M. Ferrabone, M. De La Pierre, Ph. D'Arco, et al., Int. J. Quantum Chem. 114, 1287 (2014).

45 R. Dovesi, V. R. Saunders, C. Roetti, R. Orlando, C. M. Zicovich-Wilson, F. Pascale, K. Doll, N. M. Harrison, B. Civalleri, I. J. Bush, et al., CRYSTAL14 User's Manual, Università di Torino, Torino (2014), http://www.crystal.unito.it.

46 A. Erba, M. Ferrabone, R. Orlando, and R. Dovesi, J. Comput. Chem. 34, 346 (2013).

47 K. Parlinski, Z. Q. Li, and Y. Kawazoe, Phys. Rev. Lett. 78, 4063 (1997).

48 A. Togo, F. Oba, and I. Tanaka, Phys. Rev. B 78, 134106 (2008).

49 R. E. Allen and F. W. De Wette, Phys. Rev. 179, 873 (1969).

50 L. L. Boyer, Phys. Rev. Lett. 42, 584 (1979).

51 M. F. Peintinger, D. V. Oliveira, and T. Bredow, J. Comput. Chem. 34, 451 (2013).

52 J. C. Slater, Phys. Rev. 81, 385 (1951).

53 S. H. Vosko, L. Wilk, and M. Nusair, Can. J. Phys. 58, 1200 (1980).

54 J. P. Perdew, K. Burke, and M. Ernzerhof, Phys. Rev. Lett. 77, 3865 (1996).

55 J. Perdew, J. Chevary, S. Vosko, K. Jackson, M. Pederson, D. Singh, and C. Fiolhais, Phys. Rev. B 46, 6671 (1992).

56 J. Perdew, A. Ruzsinsky, G. I. Csonka, O. A. Vydrov, G. E. Scuseria, L. A. Constantin, X. Zhou, and K. Burke, Phys. Rev. Lett. 100, 136406 (2008).

57 A. D. Becke, J. Chem. Phys. 98, 5648 (1993).

58 C. Adamo and V. Barone, J. Chem. Phys. 110, 6158 (1999).

59 S. K. Saxena and G. Shen, J. Geophys. Res. B: Solid Earth 97, 19813 (1992).
60 R. A. Robie, B. S. Hemingway, and J. R. Fisher, Tech. Rep. B - 1452, United States Geological Survey (1978).

61 B. B. Karki and R. M. Wentzcovitch, Phys. Rev. B 68, 224304 (2003).

62 O. L. Anderson, D. L. Isaak, and H. Oda, J. Geophys. Res. B: Solid Earth 96, 18037 (1991).

63 G. Fiquet, P. Richet, and G. Montagnac, Phys. Chem. Miner. 27, 103 (1999).

64 F. C. Marton and R. E. Cohen, Am. Mineral. 79, 789 (1994).

65 W. Duan, B. B. Karki, and R. M. Wentzcovitch, Am. Mineral. 84, 1961 (1999).

66 R. Caracas and R. E. Cohen, Geophys. Res. Lett. 32, L06303 (2005).

67 B. Montanari, B. Civalleri, C. M. Zicovich-Wilson, and R. Dovesi, Int. J. Quant. Chem. 106, 1703 (2006).

68 M. Iuga, G. Steinle-Neumann, and J. Meinhardt, Eur. Phys. J. B. 58, 127 (2007).

69 K. Kunc and K. Syassen, Phys. Rev. B 81, 134102 (2010).

70 D. P. Sigumonrong, D. Musici, and J. M. Schneider, Comput. Mater. Sci. 50, 1197 (2011).

71 A. Dewaele and M. Torrent, Phys. Rev. B 88, 064107 (2013).

72 R. G. Munro, J. Am. Ceram. Soc. 80, 1919 (1997).

73 G. K. White and R. B. Roberts, High Temp. High Pressures 15, 321 (1983).

74 Y. Sato and S. Akimoto, J. Appl. Phys. 50, 5285 (1979).

75 K.-D. Grevel, M. Burchard, D. W. Faßhauer, and T. Peun, J. Geophys. Res. 105, 27877 (2000).

76 L. S. Dubrovinsky, S. K. Saxena, and P. Lazor, Phys. Chem. Miner. 25, 434 (1998).

77 A. Mahmoud, A. Erba, K. Doll, and R. Dovesi, J. Chem. Phys. 140, 234703 (2014).

78 A. Erba, L. Maschio, S. Salustro, and S. Casassa, J. Chem. Phys. 134, 074502 (2011).

79 A. Erba, L. Maschio, C. Pisani, and S. Casassa, Phys. Rev. B 84, 012101 (2011).

80 V. Lacivita, A. Erba, R. Dovesi, and Ph. D’Arco, Phys. Chem. Chem. Phys. 16, 15331 (2014).

81 A. Erba, A. M. Navarrete-López, V. Lacivita, P. D’Arco, and C. M. Zicovich-Wilson, Phys. Chem. Chem. Phys. 17, 2660 (2015).

82 J. Maul, A. Erba, I. M. G. Santos, J. R. Sambrano, and R. Dovesi, J. Chem. Phys. 142, 014505 (2015).

83 T. Goto, O. L. Anderson, I. Ohno, and S. Yamamoto, J. Geophys. Res. B: Solid Earth 94, 7588 (1989). 